\title{
On a Discrete Analogue of the Two-Dimensional Toda Lattice Hierarchy
}

\author{
By
}

Satoshi Tsujimoto*

\begin{abstract}
A discrete analogue of the two-dimensional Toda lattice hierarchy, which includes the discrete analogue of the two-dimensional Toda lattice equation, is obtained by extending the theory of the Toda lattice hierarchy. Special solutions and bilinear equations for the discrete analogue of the two-dimensional Toda lattice hierarchy are presented. It is shown that the subjects originated from the Sato theory are useful for the discrete-time integrable systems.
\end{abstract}

\section{$\S 1 . \quad$ Introduction}

In early 80's, Mikio Sato constructed a remarkable theory of the soliton equations [11], [13], [14], [15]. He discovered that the $\tau$-function of the Kadomtsev-Petviashvili (KP) equation is closely related to certain algebraic identities, and found that the totality of solutions for the KP equation and its higher order equations forms an infinite dimensional Grassmann manifold. Subsequently, Ueno-Takasaki developed the Sato theory and presented the 2dimensional Toda lattice (2DTL) equation and its higher order equations [19]. Here the 2DTL equation can be regarded as a spatial discretization of the KP equation. In these theories, continuous-time soliton equations such as the KP

Communicated by M. Kashiwara. Received February 19, 2001. Revised May 7, 2001. 2000 Mathematics Subject Classification(s): 35Q51, 37K10, 37K60.

This work is supported by Grant-in-Aid for Scientific Research (No. 12640121) from the Japan Ministry of Education, Culture, Sports, Science and Technology.

* Department of Informatics and Mathematical Science, Graduate School of Engineering Science, Osaka University, Osaka 560-8531, Japan.

Present address: Department of Applied Mathematics and Physics, Graduate School of Informatics, Kyoto University, Kyoto 606-8501, Japan.

e-mail: tujimoto@i.kyoto-u.ac.jp 
equation, the 2DTL equation, have been discussed in detail, however discretetime soliton equations have not yet been fully understood.

In the last decade, discrete integrable system has been getting a lot of attention from the viewpoints of difference scheme and algorithm. For example, the discrete-time Toda lattice [7], [16] is related to the LR factorization algorithm for calculating eigenvalues of a square matrix [9], [12], [17]. Already there have been many discussions about discretizations of integrable nonlinear differential equations preserving their integrability from various points of view. Miwa revealed the link between the continuous variables $x$ and the discrete variables $m[10]$

$$
x_{j}=m_{1} \frac{\left(c_{1}\right)^{j}}{j}+m_{2} \frac{\left(c_{2}\right)^{j}}{j}+m_{3} \frac{\left(c_{3}\right)^{j}}{j}+\cdots .
$$

It is well-known that the following discrete bilinear equation

$$
\begin{aligned}
& c_{1}\left(c_{2}-c_{3}\right) \tau\left(m_{1}-c_{1}, m_{2}, m_{3}\right) \tau\left(m_{1}, m_{2}-c_{2}, m_{3}-c_{3}\right) \\
& \quad+c_{2}\left(c_{3}-c_{1}\right) \tau\left(m_{1}, m_{2}-c_{2}, m_{3}\right) \tau\left(m_{1}-c_{1}, m_{2}, m_{3}-c_{3}\right) \\
& \quad+c_{3}\left(c_{1}-c_{2}\right) \tau\left(m_{1}, m_{2}, m_{3}-c_{3}\right) \tau\left(m_{1}-c_{1}, m_{2}-c_{2}, m_{3}\right) \\
& =0
\end{aligned}
$$

where $c_{1}, c_{2}, c_{3}$ are constants related to the difference step size of the discrete independent variables [8], [10], can be derived from the bilinear form of the KP equation

$$
\left(D_{x_{1}}^{4}-4 D_{x_{1}} D_{x_{3}}+3 D_{x_{2}}^{2}\right) \tau \cdot \tau=0,
$$

and its higher order equations by introducing infinitely many discrete-time variables $m=\left(m_{1}, m_{2}, m_{3}, \ldots\right)[10]$. The operator $D$ is defined as

$$
D_{x}^{m} a(x) \cdot b(x)=\left.\frac{\partial}{\partial x^{\prime}} a\left(x+x^{\prime}\right) b\left(x-x^{\prime}\right)\right|_{x^{\prime}=0} .
$$

A special solution of Casorati determinant form to Eqs. (1) and (2) is expressed as

$$
\tau=\operatorname{det}\left|f_{i}(s+j-1, x, m)\right|_{1 \leq i, j \leq N},
$$

where all $f_{i}$, which are functions of $s, x=\left(x_{1}, x_{2}, x_{3}, \ldots\right), m=\left(m_{1}, m_{2}, m_{3}, \ldots\right)$, satisfy the following linear equations

$$
\begin{aligned}
\frac{\partial}{\partial x_{j}} f_{i}(s, x, m) & =f_{i}(s+j, x, m), \\
\frac{1}{c_{j}}\left(1-\exp \left(-\frac{\partial}{\partial m_{j}}\right)\right) f_{i}(s, x, m) & =f_{i}(s+1, x, m),
\end{aligned}
$$


for $i=1,2, \ldots, N$ and $j=1,2, \ldots$ Hereafter the unshifted independent variables are often omitted and only the shifted variables are written down explicitly. For example, Eq. (5) is rewritten as $\left(f_{i}\left(s ; m_{j}\right)-f_{i}\left(s ; m_{j}-1\right)\right) / c_{j}=$ $f_{i}\left(s+1 ; m_{j}\right)$. Furthermore, Date, Jimbo and Miwa developed Sato's idea as a method for generating discrete soliton equations from the bilinear differential equations [1], [2], [3], [4], [5], [6]. Their method is based on the "transformation groups" theory developed by themselves and their collaborators. In this paper, we will introduce sets of infinite number of discrete variables suitable for the discrete analogues of the 2DTL hierarchy.

The purpose of this paper is to present a discrete analogue of the 2DTL hierarchy which includes the discrete-time 2DTL equation [9] by extending the theory of the 2DTL hierarchy, and to investigate special solutions expressed by Casorati determinants and a bilinear expression of the discrete analogue of the 2DTL hierarchy. A part of this paper's result was announced in [18]. The key is a discrete analogue of the Sato equation introduced in Section 3.

This paper is planned as follows. We will build up a theory of the discreteanalogue of the 2DTL hierarchy. Our theory is much indebted to the results of Ueno-Takasaki [19]. In Section 2, we give a brief review of the continuoustime 2DTL hierarchy. In Section 3, we present suitable Sato type equations and obtain the Lax type equations and the Zakharov-Shabat type equations of the discrete analogue of the 2DTL hierarchy. In Section 4, we discuss special solutions of Casorati determinant form to the discrete Sato equations by considering a simultaneous equation. In Section 5, we derive a part of infinitely many bilinear equations explicitly. In Section 6 , an infinite matrix expression of the hierarchy of the discrete-time 2DTL equations is given in a discrete Lax form. The last section is devoted to concluding remarks.

The author would like to thank Professor R. Hirota and Y. Nakamura for useful discussions and continuous encouragements.

\section{$\S 2 . \quad$ Toda Lattice Hierarchy}

In this section, we give a brief review of the 2DTL hierarchy proposed by Ueno-Takasaki [19]. First of all, we start from the following difference operators

$$
\begin{aligned}
W^{(\infty)} & =1+w_{1}^{(\infty)} e^{-\partial_{s}}+w_{2}^{(\infty)} e^{-2 \partial_{s}}+w_{3}^{(\infty)} e^{-3 \partial_{s}}+\cdots, \\
W^{(0)} & =w_{0}^{(0)}+w_{1}^{(0)} e^{\partial_{s}}+w_{2}^{(0)} e^{2 \partial_{s}}+w_{3}^{(0)} e^{3 \partial_{s}}+\cdots,
\end{aligned}
$$

where $\partial_{s}$ denotes $\partial / \partial s, e^{\partial_{s}}$ is a shift operator, and the coefficients of $e^{j \partial_{s}}$ of $L$ and $M$ are functions of $s, x=\left(x_{1}, x_{2}, \ldots\right), y=\left(y_{1}, y_{2}, \ldots\right)$, namely 
$w_{1}^{(\infty)}=w_{1}^{(\infty)}(s, x, y)$, and so on. Here $x$ and $y$ indicate continuous independent variables, and $s$ denotes a discrete independent variable where $s$ is sometimes called a discrete spatial variable. These operators $W^{(\infty)}$ and $W^{(0)}$ play an important role not only in the 2DTL hierarchy, but also in the discrete analogue of the 2DTL hierarchy.

Let $L$ and $M$ be difference operators defined by

$$
\begin{aligned}
L & =W^{(\infty)} e^{\partial_{s}} W^{(\infty)-1} \\
& =e^{\partial_{s}}+u_{1}+u_{2} e^{-\partial_{s}}+u_{3} e^{-2 \partial_{s}}+\cdots, \\
M & =W^{(0)} e^{-\partial_{s}} W^{(0)-1} \\
& =v_{0} e^{-\partial_{s}}+v_{1}+v_{2} e^{\partial_{s}}+v_{3} e^{2 \partial_{s}}+\cdots .
\end{aligned}
$$

From parts of the operators $L$ and $M$, the operators $B_{n}, C_{n}$ are defined by

$$
\begin{aligned}
& B_{n}=\left(L^{n}\right)_{+}, \\
& C_{n}=\left(M^{n}\right)_{-},
\end{aligned}
$$

where the suffices + and - mean parts which do not include $e^{-\partial_{s}}, e^{-2 \partial_{s}}, \ldots$, and $e^{0 \partial_{s}}, e^{\partial_{s}}, e^{2 \partial_{s}}, \ldots$, respectively. We introduce time evolution equations of $W^{(\infty)}$ and $W^{(0)}$, the Sato type equations of the 2DTL hierarchy, by

$$
\begin{aligned}
\frac{\partial W^{(\infty)}}{\partial x_{n}} & =B_{n} W^{(\infty)}-W^{(\infty)} e^{n \partial_{s}}, \\
\frac{\partial W^{(\infty)}}{\partial y_{n}} & =C_{n} W^{(\infty)}-W^{(\infty)} e^{-n \partial_{s}}, \\
\frac{\partial W^{(0)}}{\partial x_{n}} & =B_{n} W^{(0)}-W^{(0)} e^{n \partial_{s}}, \\
\frac{\partial W^{(0)}}{\partial y_{n}} & =C_{n} W^{(0)}-W^{(0)} e^{-n \partial_{s}}
\end{aligned}
$$

for $n=1,2,3, \ldots$.

From Eqs. (7) and (8), the 2DTL hierarchy is formulated as a system of infinite number of the Lax type equations

$$
\begin{aligned}
& \frac{\partial L}{\partial x_{n}}=\left[B_{n}, L\right], \\
& \frac{\partial L}{\partial y_{n}}=\left[C_{n}, L\right], \\
& \frac{\partial M}{\partial x_{n}}=\left[B_{n}, M\right], \\
& \frac{\partial M}{\partial y_{n}}=\left[C_{n}, M\right],
\end{aligned}
$$


or equivalently, the Zakharov-Shabat type equations

$$
\begin{aligned}
& \frac{\partial B_{m}}{\partial x_{n}}-\frac{\partial B_{n}}{\partial x_{m}}+\left[B_{m}, B_{n}\right]=0, \\
& \frac{\partial C_{m}}{\partial y_{n}}-\frac{\partial C_{n}}{\partial y_{m}}+\left[C_{m}, C_{n}\right]=0, \\
& \frac{\partial B_{m}}{\partial y_{n}}-\frac{\partial C_{n}}{\partial x_{m}}+\left[B_{m}, C_{n}\right]=0,
\end{aligned}
$$

which gives an infinite number of nonlinear differential-difference equations. The bracket $[X, Y]$ means $X Y-Y X$. From (9) or (10) by setting $m=n=1$, we obtain the 2DTL equation

$$
\begin{aligned}
& \frac{\partial}{\partial x_{1}} v_{0}(s)=v_{0}(s)\left(u_{1}(s)-u_{1}(s-1)\right), \\
& \frac{\partial}{\partial y_{1}} u_{1}(s)=-v_{0}(s+1)+v_{0}(s),
\end{aligned}
$$

as a simple example.

\section{§3. Discrete Analogue of Toda Lattice Hierarchy}

A natural extension of the 2DTL theory will be made in this section. It is shown that discrete analogues of the Sato equations, the Lax type equations and the Zakharov-Shabat type equations for the 2DTL hierarchy can be derived from suitable difference operators in which we introduce infinite number of discrete variables. We give some explicit examples including the discrete 2DTL equation [9] as the simplest nontrivial one.

Using the difference operators $W^{(\infty)}, W^{(0)}$ which are introduced in the preceding section, we define the following difference operators $\mathcal{L}_{n}$ and $\mathcal{M}_{n}$ by

$$
\begin{aligned}
\mathcal{L}_{n} & =e^{\partial_{k_{n}}} W^{(\infty)} e^{-\partial_{k_{n}}} e^{n \partial_{s}} W^{(\infty)-1} \\
& =e^{n \partial_{s}}+u_{1}^{(n)} e^{(n-1) \partial_{s}}+u_{2}^{(n)} e^{(n-2) \partial_{s}}+u_{3}^{(n)} e^{(n-3) \partial_{s}}+\cdots \\
\mathcal{M}_{n} & =e^{\partial_{l_{n}}} W^{(0)} e^{-\partial_{l_{n}}} e^{-n \partial_{s}} W^{(0)-1} \\
& =v_{0}^{(n)} e^{-n \partial_{s}}+v_{1}^{(n)} e^{(1-n) \partial_{s}}+v_{2}^{(n)} e^{(2-n) \partial_{s}}+v_{3}^{(n)} e^{(3-n) \partial_{s}}+\cdots
\end{aligned}
$$

where the coefficients of $e^{j \partial_{s}}$ in $\mathcal{L}_{n}$ and $\mathcal{M}_{n}$ are functions of $s, x=\left(x_{1}, x_{2}, \ldots\right)$, $y=\left(y_{1}, y_{2}, \ldots\right)$, and also $k=\left(k_{1}, k_{2}, \ldots\right), l=\left(l_{1}, l_{2}, \ldots\right)$, namely $u_{j}^{(n)}=$ $u_{j}^{(n)}(s, x, y, k, l), v_{j}^{(n)}=v_{j}^{(n)}(s, x, y, k, l)$. Here $s, k, l$ denote discrete independent variables. 
Let us suppose that $W^{(\infty)}$ and $W^{(0)}$ satisfy discrete analogues of the Sato equations

$$
\begin{aligned}
\Delta_{k_{n}}\left(W^{(\infty)}\right) & =\mathcal{B}_{n} W^{(\infty)}-e^{\partial_{k_{n}}} W^{(\infty)} e^{-\partial_{k_{n}}} e^{n \partial_{s}}, \\
\Delta_{l_{n}}\left(W^{(\infty)}\right) & =\mathcal{C}_{n} W^{(\infty)}-e^{\partial_{l_{n}}} W^{(\infty)} e^{-\partial_{l_{n}}} e^{-n \partial_{s}}, \\
\Delta_{k_{n}}\left(W^{(0)}\right) & =\mathcal{B}_{n} W^{(0)}-e^{\partial_{k_{n}}} W^{(0)} e^{-\partial_{k_{n}}} e^{n \partial_{s}}, \\
\Delta_{l_{n}}\left(W^{(0)}\right) & =\mathcal{C}_{n} W^{(0)}-e^{\partial_{l_{n}}} W^{(0)} e^{-\partial_{l_{n}}} e^{-n \partial_{s}}
\end{aligned}
$$

where the difference operators $\mathcal{B}_{n}, \mathcal{C}_{n}$ are defined by

$$
\begin{aligned}
& \mathcal{B}_{n}=\left(\mathcal{L}_{n}\right)_{+}, \\
& \mathcal{C}_{n}=\left(\mathcal{M}_{n}\right)_{-} .
\end{aligned}
$$

Here we also define the difference operators $\Delta_{k_{n}}$ and $\Delta_{l_{n}}$ by

$$
\begin{aligned}
\Delta_{k_{n}} & =\frac{1}{a_{n}}\left(e^{\partial_{k_{n}}}-1\right), \\
\Delta_{l_{n}} & =\frac{1}{b_{n}}\left(e^{\partial_{l_{n}}}-1\right),
\end{aligned}
$$

where $a_{1}, a_{2}, \ldots, b_{1}, b_{2}, \ldots$ are difference step sizes for the discrete independent variables $k_{1}, k_{2}, \ldots, l_{1}, l_{2}, \ldots$, respectively.

Now let us derive a discrete analogue of the Lax equations (9) from Eqs. (11) and (12). If we notice that

$$
\begin{aligned}
\Delta_{k_{n}}\left(W^{(\infty)} W^{(\infty)-1}\right) & =\Delta_{k_{n}}\left(W^{(\infty)}\right) W^{(\infty)-1}+e^{\partial_{k_{n}}} W^{(\infty)} e^{-\partial_{k_{n}}} \Delta_{k_{n}}\left(W^{(\infty)-1}\right) \\
& =0
\end{aligned}
$$

we get

$$
\begin{aligned}
\Delta_{k_{n}}\left(W^{(\infty)-1}\right) & =-e^{\partial_{k_{n}}} W^{(\infty)-1} e^{-\partial_{k_{n}}} \Delta_{k_{n}}\left(W^{(\infty)}\right) W^{(\infty)-1} \\
& =-e^{\partial_{k_{n}}} W^{(\infty)-1} e^{-\partial_{k_{n}}} \mathcal{B}_{n}+e^{n \partial_{s}} W^{(\infty)-1}
\end{aligned}
$$

By employing the equation above, a discrete Lax equation corresponding to the 
Lax equation (9a) is given as follows

$$
\begin{aligned}
& \Delta_{k_{n}}\left(\mathcal{L}_{m}\right)=e^{\partial_{k_{m}}} \Delta_{k_{n}}\left(W^{(\infty)}\right) e^{-\partial_{k_{m}}} e^{m \partial_{s}} W^{(\infty)-1} \\
& +e^{\partial_{k_{m}}+\partial_{k_{n}}} W^{(\infty)} e^{-\partial_{k_{m}}-\partial_{k_{n}}} e^{m \partial_{s}} \Delta_{k_{n}}\left(W^{(\infty)-1}\right) \\
& =e^{\partial_{k_{m}}}\left(\mathcal{B}_{n} W^{(\infty)}-e^{\partial_{k_{n}}} W^{(\infty)} e^{-\partial_{k_{n}}} e^{n \partial_{s}}\right) e^{-\partial_{k_{m}}} e^{m \partial_{s}} W^{(\infty)-1} \\
& +e^{\partial_{k_{m}}+\partial_{k_{n}}} W^{(\infty)} e^{-\partial_{k_{m}}-\partial_{k_{n}}+} e^{m \partial_{s}}\left(e^{n \partial_{s}} W^{(\infty)-1}-e^{\partial_{k_{n}}} W^{(\infty)-1} e^{-\partial_{k_{n}}} \mathcal{B}_{n}\right) \\
& =e^{\partial_{k_{m}}} \mathcal{B}_{n} W^{(\infty)} e^{-\partial_{k_{m}}} e^{m \partial_{s}} W^{(\infty)-1} \\
& -e^{\partial_{k_{m}}+\partial_{k_{n}}} W^{(\infty)} e^{-\partial_{k_{m}}} e^{m \partial_{s}} W^{(\infty)-1} e^{-\partial_{k_{n}}} \mathcal{B}_{n} \\
& =e^{\partial_{k_{m}}} \mathcal{B}_{n} e^{-\partial_{k_{m}}} \mathcal{L}_{m}-e^{\partial_{k_{n}}} \mathcal{L}_{m} e^{-\partial_{k_{n}}} \mathcal{B}_{n} \text {. }
\end{aligned}
$$

As a consequence of the same procedure, we obtain discrete analogues of the Lax equations (9)

$$
\begin{aligned}
\Delta_{k_{n}}\left(\mathcal{L}_{m}\right) & =\left[\mathcal{B}_{n}, \mathcal{L}_{m}\right]_{k_{m}, k_{n}}, \quad m \neq n, \\
\Delta_{l_{n}}\left(\mathcal{L}_{m}\right) & =\left[\mathcal{C}_{n}, \mathcal{L}_{m}\right]_{k_{m}, l_{n}}, \\
\Delta_{k_{n}}\left(\mathcal{M}_{m}\right) & =\left[\mathcal{B}_{n}, \mathcal{M}_{m}\right]_{l_{m}, k_{n}}, \\
\Delta_{l_{n}}\left(\mathcal{M}_{m}\right) & =\left[\mathcal{C}_{n}, \mathcal{M}_{m}\right]_{l_{m}, l_{n}}, \quad m \neq n, \\
0 & =\left[\mathcal{L}_{m}, \mathcal{L}_{n}\right]_{k_{n}, k_{m}}, \\
0 & =\left[\mathcal{M}_{m}, \mathcal{M}_{n}\right]_{l_{n}, l_{m}},
\end{aligned}
$$

where $[X, Y]_{x, y}$ indicates $e^{\partial x} X e^{-\partial x} Y-e^{\partial y} Y e^{-\partial y} X$. Eqs. (13e) and (13f) are readily verified from the definition of $\mathcal{L}_{m}, \mathcal{M}_{m}$.

The equations (13) can be expressed equivalently as

$$
\begin{aligned}
\Delta_{k_{m}}\left(\mathcal{B}_{n}\right)-\Delta_{k_{n}}\left(\mathcal{B}_{m}\right)+\left[\mathcal{B}_{n}, \mathcal{B}_{m}\right]_{k_{m}, k_{n}} & =0, \\
\Delta_{l_{m}}\left(\mathcal{C}_{n}\right)-\Delta_{l_{n}}\left(\mathcal{C}_{m}\right)+\left[\mathcal{C}_{n}, \mathcal{C}_{m}\right]_{l_{m}, l_{n}} & =0, \\
\Delta_{l_{m}}\left(\mathcal{B}_{n}\right)-\Delta_{k_{n}}\left(\mathcal{C}_{m}\right)+\left[\mathcal{B}_{n}, \mathcal{C}_{m}\right]_{l_{m}, k_{n}} & =0,
\end{aligned}
$$

which are discrete analogues of the Zakharov-Shabat equations (10).

Let us give a proof of (14). We can derive Eq. (14a) from Eqs. (13a) and (13e) as the following way

$$
\begin{aligned}
\Delta_{k_{m}}\left(\mathcal{L}_{n}\right)-\Delta_{k_{n}}\left(\mathcal{L}_{m}\right) & =\left[\mathcal{B}_{m}, \mathcal{L}_{n}\right]_{k_{n}, k_{m}}-\left[\mathcal{B}_{n}, \mathcal{L}_{m}\right]_{k_{m}, k_{n}} \\
& =\left[\mathcal{L}_{m}-\underline{\mathcal{L}}_{m}, \mathcal{L}_{n}\right]_{k_{n}, k_{m}}-\left[\mathcal{B}_{n}, \mathcal{L}_{m}\right]_{k_{m}, k_{n}} \\
& =\left[\mathcal{L}_{n}, \mathcal{L}_{m}\right]_{k_{m}, k_{n}}-\left[\mathcal{B}_{n}, \mathcal{L}_{m}\right]_{k_{m}, k_{n}} \\
& =\left[\mathcal{B}_{n}+\underline{\mathcal{L}}_{n}, \underline{\mathcal{L}}_{m}\right]_{k_{m}, k_{n}}-\left[\mathcal{B}_{n}, \mathcal{B}_{m}+\underline{\mathcal{L}}_{m}\right]_{k_{m}, k_{n}} \\
& =\left[\underline{\mathcal{L}}_{n}, \underline{\mathcal{L}}_{m}\right]_{k_{m}, k_{n}}-\left[\mathcal{B}_{n}, \mathcal{B}_{m}\right]_{k_{m}, k_{n}},
\end{aligned}
$$


where $\underline{\mathcal{L}}_{m}$ denotes $\left(\mathcal{L}_{m}\right)_{-}$. Then we take the plus part of the equation above, and obtain (14a).

Next we can obtain Eq. (14c) from Eqs. (13d) and (13f) as follows. Note that

$$
\begin{aligned}
\Delta_{l_{m}}\left(\mathcal{M}_{n}\right)-\Delta_{l_{n}}\left(\mathcal{M}_{m}\right) & =\left[\mathcal{C}_{m}, \mathcal{M}_{n}\right]_{l_{n}, l_{m}}-\left[\mathcal{C}_{n}, \mathcal{M}_{m}\right]_{l_{m}, l_{n}} \\
& =\left[\mathcal{M}_{m}-\underline{\mathcal{M}}_{m}, \mathcal{M}_{n}\right]_{l_{n}, l_{m}}-\left[\mathcal{C}_{n}, \mathcal{M}_{m}\right]_{l_{m}, l_{n}} \\
& =\left[\mathcal{M}_{n}, \underline{\mathcal{M}}_{m}\right]_{l_{m}, l_{n}}-\left[\mathcal{C}_{n}, \mathcal{M}_{m}\right]_{l_{m}, l_{n}} \\
& =\left[\mathcal{C}_{n}+\underline{\mathcal{M}}_{n}, \underline{\mathcal{M}}_{m}\right]_{l_{m}, l_{n}}-\left[\mathcal{C}_{n}, \mathcal{C}_{m}+\underline{\mathcal{M}}_{m}\right]_{l_{m}, l_{n}} \\
& =\left[\underline{\mathcal{M}}_{n}, \underline{\mathcal{M}}_{m}\right]_{l_{m}, l_{n}}-\left[\mathcal{C}_{n}, \mathcal{C}_{m}\right]_{l_{m}, l_{n}}
\end{aligned}
$$

Then we take the plus part of the equation above, and obtain (14c).

Finally we show that (14b) is given from (13). Eqs. (13b) and (13c)

$$
\begin{aligned}
\Delta_{l_{n}} \mathcal{L}_{m} & =\left[\mathcal{C}_{n}, \mathcal{L}_{m}\right]_{k_{m}, l_{n}}, \\
\Delta_{k_{n}} \mathcal{M}_{m} & =\left[\mathcal{B}_{n}, \mathcal{M}_{m}\right]_{l_{m}, k_{n}}
\end{aligned}
$$

are rewritten as

$$
\begin{aligned}
\Delta_{l_{n}}\left(\mathcal{B}_{m}\right)-\left[\mathcal{C}_{n}, \mathcal{B}_{m}^{(k)}\right]_{k_{m}, l_{n}} & =-\Delta_{l_{n}}\left(\underline{\mathcal{L}}_{m}\right)+\left[\mathcal{C}_{n}, \underline{\mathcal{L}}_{m}\right]_{k_{m}, l_{n}} \\
\Delta_{k_{n}}\left(\overline{\mathcal{M}}_{m}\right)-\left[\mathcal{B}_{n}, \overline{\mathcal{M}}_{m}\right]_{l_{m}, k_{n}} & =-\Delta_{k_{n}}\left(\mathcal{C}_{m}\right)+\left[\mathcal{B}_{n}, \mathcal{C}_{m}\right]_{l_{m}, k_{n}}
\end{aligned}
$$

where $\overline{\mathcal{M}}_{m}$ denotes $\left(\mathcal{M}_{m}\right)_{+}$, respectively. By summing Eqs. (15), we have

$$
\begin{aligned}
\Delta_{l_{m}}\left(\mathcal{B}_{n}\right)+\Delta_{k_{n}}\left(\overline{\mathcal{M}}_{m}\right) & -\left[\mathcal{B}_{n}, \overline{\mathcal{M}}_{m}\right]_{l_{m}, k_{n}} \\
& =-\Delta_{k_{n}}\left(\mathcal{C}_{m}\right)-\Delta_{l_{m}}\left(\underline{\mathcal{L}}_{n}\right)+\left[\mathcal{C}_{m}, \underline{\mathcal{L}}_{n}\right]_{k_{n}, l_{m}}
\end{aligned}
$$

Taking the plus part of the equation above, we find that

$$
\Delta_{l_{m}}\left(\mathcal{B}_{n}\right)+\Delta_{k_{n}}\left(\overline{\mathcal{M}}_{m}\right)-\left[\mathcal{B}_{n}, \overline{\mathcal{M}}_{m}\right]_{l_{m}, k_{n}}=0 .
$$

Substituting (15a) into (16), we obtain (14b).

From Eqs. (13) or (14), we obtain an infinite number of nonlinear difference equations that we name the discrete analogue of the Toda lattice hierarchy. For example, from (14c) by setting $m=n=1$, we obtain

$$
\left\{\begin{array}{c}
\Delta_{l_{1}} u_{1}^{(1)}\left(s ; k_{1}, l_{1}\right)+v_{0}^{(1)}\left(s+1 ; k_{1}, l_{1}\right) \\
-v_{0}^{(1)}\left(s ; k_{1}+1, l_{1}\right)=0 \\
-\Delta_{k_{1}} v_{0}^{(1)}\left(s ; k_{1}, l_{1}\right)+u_{1}^{(1)}\left(s ; k_{1}, l_{1}+1\right) v_{0}^{(1)}\left(s ; k_{1}, l_{1}\right) \\
-v_{0}^{(1)}\left(s ; k_{1}+1, l_{1}\right) u_{1}^{(1)}\left(s-1 ; k_{1}, l_{1}\right)=0
\end{array},\right.
$$


which is just the discrete 2DTL equation [9]. Moreover, we derive from (14c) by setting $m=1, n=2$

$$
\left\{\begin{array}{c}
\Delta_{l_{1}} u_{1}^{(2)}\left(s ; k_{2}, l_{1}\right)+v_{0}^{(1)}\left(s+2 ; k_{2}, l_{1}\right)-v_{0}^{(1)}\left(s ; k_{2}+1, l_{1}\right)=0 \\
\Delta_{l_{1}} v_{2}^{(2)}\left(s ; k_{2}, l_{1}\right)+u_{1}^{(2)}\left(s ; k_{2}, l_{1}+1\right) v_{0}^{(1)}\left(s+1 ; k_{2}, l_{1}\right) \\
-v_{0}^{(1)}\left(s ; k_{2}+1, l_{1}\right) u_{1}^{(2)}\left(s-1 ; k_{2}, l_{1}\right)=0 \\
-\Delta_{k_{2}} v_{0}^{(1)}\left(s ; k_{2}, l_{1}\right)+u_{2}^{(2)}\left(s ; k_{2}, l_{1}+1\right) v_{0}^{(1)}\left(s ; k_{2}, l_{1}\right) \\
-v_{0}^{(1)}\left(s ; k_{2}+1, l_{1}\right) u_{2}^{(2)}\left(s-1 ; k_{2}, l_{1}\right)=0
\end{array} .\right.
$$

An infinite number of differential-difference equations with respect to the variables $x, y, k, l$ is also obtained from Eqs. (8) and (12) as

$$
\begin{aligned}
& \frac{\partial \mathcal{B}_{n}}{\partial x_{m}}-\left(\Delta_{k_{n}} B_{m}\right)=\left(e^{\partial_{k_{n}}} B_{m}\right) \mathcal{B}_{n}-\mathcal{B}_{n} B_{m}, \\
& \frac{\partial \mathcal{B}_{n}}{\partial y_{m}}-\left(\Delta_{k_{n}} C_{m}\right)=\left(e^{\partial_{k_{n}}} C_{m}\right) \mathcal{B}_{n}-\mathcal{B}_{n} C_{m}, \\
& \frac{\partial \mathcal{C}_{n}}{\partial x_{m}}-\left(\Delta_{l_{n}} B_{m}\right)=\left(e^{\partial_{l_{n}}} B_{m}\right) \mathcal{C}_{n}-\mathcal{C}_{n} B_{m}, \\
& \frac{\partial \mathcal{C}_{n}}{\partial y_{m}}-\left(\Delta_{l_{n}} C_{m}\right)=\left(e^{\partial_{l_{n}}} C_{m}\right) \mathcal{C}_{n}-\mathcal{C}_{n} C_{m} .
\end{aligned}
$$

Some examples of integrable differential-difference equations are then

$$
\begin{gathered}
\left\{\begin{array}{c}
u_{1}^{(1)}\left(s ; k_{1}\right)+u_{1}\left(s+1 ; k_{1}\right)=u_{1}^{(1)}\left(s+1 ; k_{1}\right)+u_{1}\left(s ; k_{1}+1\right) \\
\frac{\partial}{\partial x_{1}} u_{1}^{(1)}\left(s ; k_{1}\right)-\frac{u_{1}\left(s ; k_{1}+1\right)-u_{1}\left(s ; k_{1}\right)}{a_{1}} \\
=u_{1}^{(1)}\left(s ; k_{1}\right)\left(u_{1}\left(s ; k_{1}+1\right)-u_{1}\left(s ; k_{1}\right)\right)
\end{array}\right. \\
\left\{\begin{array}{c}
\frac{\partial}{\partial y_{1}} u_{1}^{(1)}\left(s ; k_{1}\right)=v_{0}\left(s ; k_{1}+1\right)-v_{0}\left(s+1 ; k_{1}\right) \\
\frac{v_{0}\left(s ; k_{1}+1\right)-v_{0}\left(s ; k_{1}\right)}{a_{1}}=v_{0}\left(s ; k_{1}\right) u_{1}^{(1)}\left(s ; k_{1}\right)-v_{0}\left(s ; k_{1}+1\right) u_{1}^{(1)}\left(s-1 ; k_{1}\right)
\end{array}\right. \\
\left\{\begin{array}{c}
\frac{\partial}{\partial x_{1}} \log v_{0}^{(1)}\left(s ; l_{1}\right)=u_{1}\left(s ; l_{1}+1\right)-u_{1}\left(s-1 ; l_{1}\right) \\
\frac{u_{1}\left(s ; l_{1}+1\right)-u_{1}\left(s ; l_{1}\right)}{b_{1}}=v_{0}^{(1)}\left(s ; l_{1}\right)-v_{0}^{(1)}\left(s-1 ; l_{1}\right)
\end{array}\right. \\
\left\{\begin{array}{c}
\frac{\partial}{\partial y_{1}} v_{0}^{(1)}\left(s ; l_{1}\right)=\frac{v_{0}\left(s ; l_{1}+1\right)-v_{0}\left(s ; l_{1}\right)}{b_{1}} \\
v_{0}\left(s ; l_{1}+1\right)-v_{0}^{(1)}\left(s-1 ; l_{1}\right)=v_{0}^{(1)}\left(s ; l_{1}\right)-v_{0}\left(s-1 ; l_{1}\right)
\end{array}\right.
\end{gathered}
$$




\section{$\S 4$. Solutions of Casorati Determinant Form}

In this section, we give special solutions of Casorati determinant form to the discrete analogue of the 2DTL hierarchy, originally proposed by UenoTakasaki [19].

We first consider a formal difference operator $W_{N}$, which depends on $s, x=$ $\left(x_{1}, x_{2}, \ldots\right), y=\left(y_{1}, y_{2}, \ldots\right), k=\left(k_{1}, k_{2}, \ldots\right)$, and $l=\left(l_{1}, l_{2}, \ldots\right)$, defined by

$$
W_{N}(s, x, y, k, l)=e^{N \partial_{s}}+w_{1} e^{(N-1) \partial_{s}}+w_{2} e^{(N-2) \partial_{s}}+\cdots+w_{N} .
$$

The operator $W_{N}$ is determined by the simultaneous difference equation

$$
W_{N}(s, x, y, k, l) f_{j}(s, x, y, k, l)=0
$$

for $j=1, \ldots, N$, where $f_{j}$ are the linearly independent functions in $s$. Namely, if we give a set of functions $\left\{f_{j}\right\}_{j=1,2, \ldots, N}$, the dependent variables $w_{j}$ is expressed as

$$
w_{j}=-\frac{|f(s), \ldots, f(s+N-j-1), f(s+N), f(s+N-j+1), \ldots, f(s+N-1)|}{|f(s), \ldots, f(s+N-1)|}
$$

for $j=1, \ldots, N$, where we have introduced a new notation of determinant for simplicity,

$$
\begin{aligned}
|f(s), f(s+1), \cdots, f(s+N-1)| & \\
& \equiv \operatorname{det}\left|\begin{array}{cccc}
f_{1}(s) & f_{1}(s+1) & \cdots & f_{1}(s+N-1) \\
f_{2}(s) & f_{2}(s+1) & \cdots & f_{2}(s+N-1) \\
\vdots & \vdots & \ddots & \vdots \\
f_{N}(s) & f_{N}(s+1) & \cdots & f_{N}(s+N-1)
\end{array}\right| .
\end{aligned}
$$

The determinant $|f(s), \cdots, f(s+N-1)|$ is called a Casorati determinant which is a discrete analogue of Wronskian.

Let us define $(x, y, k, l)$-dependence of $f_{j}(s, x, y, k, l)$ as

$$
\begin{aligned}
\frac{\partial f_{j}(s)}{\partial x_{n}} & =f_{j}(s+n), \\
\frac{\partial f_{j}(s)}{\partial y_{n}} & =f_{j}(s-n), \\
\Delta_{k_{n}} f_{j}(s) & =f_{j}(s+n), \\
\Delta_{l_{n}} f_{j}(s) & =f_{j}(s-n),
\end{aligned}
$$


for $j=1,2, \ldots, N$ and $n=1,2, \ldots$.

Under the above conditions, one can show that the difference operator $W_{N}$ satisfies the following equations,

$$
\begin{aligned}
\frac{\partial W_{N}}{\partial x_{n}} & =B_{n} W_{N}-W_{N} e^{n \partial_{s}}, \\
\frac{\partial W_{N}}{\partial y_{n}} & =C_{n} W_{N}-W_{N} e^{-n \partial_{s}}, \\
\Delta_{k_{n}}\left(W_{N}\right) & =\mathcal{B}_{n} W_{N}-e^{\partial_{k_{n}}} W_{N} e^{-\partial_{k_{n}}} e^{n \partial_{s}}, \\
\Delta_{l_{n}}\left(W_{N}\right) & =\mathcal{C}_{n} W_{N}-e^{\partial_{l_{n}}} W_{N} e^{-\partial_{l_{n}}} e^{-n \partial_{s}},
\end{aligned}
$$

where the operators $B_{n}, C_{n}, \mathcal{B}_{n}, \mathcal{C}_{n}$ satisfy

$$
\begin{aligned}
B_{n}=\left(B_{n}\right)_{+}, & C_{n}=\left(C_{n}\right)_{-}, \\
\mathcal{B}_{n}=\left(\mathcal{B}_{n}\right)_{+}, & \mathcal{C}_{n}=\left(\mathcal{C}_{n}\right)_{-} .
\end{aligned}
$$

To prove Eqs. (22), we use the following lemma. (A proof of Eqs. (22a) and $(22 \mathrm{~b})$ can be found in [19].)

Lemma 4.1 ([19]). For any difference operator $U=u_{0}+u_{1} e^{\partial_{s}}+$ $u_{2} e^{2 \partial_{s}}+\cdots$, there exist two operators $Q$ and $R$ uniquely such that

$$
\left\{\begin{array}{l}
U=Q W_{N}+R, \\
Q=\sum_{j \geq 0} q_{j}(s) e^{j \partial_{s}}, \quad R=\sum_{j=0}^{N-1} r_{j}(s) e^{j \partial_{s}} .
\end{array}\right.
$$

Similarly, for any difference operator $U^{\prime}=u_{0}^{\prime}+u_{1}^{\prime} e^{-\partial_{s}}+u_{2}^{\prime} e^{-2 \partial_{s}}+\cdots$, there exist two operators $Q^{\prime}$ and $R^{\prime}$ uniquely such that

$$
\left\{\begin{array}{l}
U^{\prime}=Q^{\prime} W_{N} e^{-N \partial_{s}}+R^{\prime}, \\
Q^{\prime}=\sum_{j \leq 0} q_{j}^{\prime}(s) e^{j \partial_{s}}, \quad R^{\prime}=\sum_{j=1-N}^{0} r_{j}^{\prime}(s) e^{j \partial_{s}} .
\end{array}\right.
$$

We first prove Eq. (22c). By using a difference analogue of the Leibniz rule

$$
\Delta_{n}(f(n) g(n))=\Delta_{n}(f(n)) g(n)+f(n+1) \Delta_{n}(g(n)),
$$

we can find

$$
\left(\Delta_{k_{n}}\left(W_{N}\right)+e^{\partial_{k_{n}}} W_{N} e^{\partial_{-k_{n}}} e^{n \partial_{s}}\right) f_{n}=0 \quad(n=1, \ldots, N)
$$


from Eq. (19). Lemma 4.1 implies that there exist certain difference operators $\mathcal{B}_{n}$, and $\mathcal{R}_{n}$ of the form

$$
\begin{aligned}
\mathcal{B}_{n} & =\sum_{j \geq 0} \beta_{j}(s, x, y, k, l) e^{j \partial_{s}}, \\
\mathcal{R}_{n} & =\sum_{j=0}^{N-1} \gamma_{j}(s, x, y, k, l) e^{j \partial_{s}}
\end{aligned}
$$

such that

$$
\Delta_{k_{n}}\left(W_{N}\right)+e^{\partial_{k_{n}}} W_{N} e^{-\partial_{k_{n}}} e^{n \partial_{s}}=\mathcal{B}_{n} W_{N}+\mathcal{R}_{n}
$$

Then we get

$$
\left(\Delta_{k_{n}}\left(W_{N}\right)+e^{\partial_{k_{n}}} W_{N} e^{-\partial_{k_{n}}} e^{n \partial_{s}}\right) f_{j}=\left(\mathcal{B}_{n} W_{N}+\mathcal{R}_{n}\right) f_{j}=0
$$

and

$$
\mathcal{R}_{n} f_{j}=0
$$

for $j=1, \ldots, N$. We conclude that $\mathcal{R}_{n}=0$, since $f_{j}$ are the linearly independent functions in $s$. Hence Eq. (22c) holds. The proof of Eqs. (22a), (22b) and (22d) are given in the same procedure.

From Eqs. (22), we find that the difference operators $B_{n}, C_{n}, \mathcal{B}_{n}, \mathcal{C}_{n}$ are written in term of $W_{N}$ as

$$
\begin{aligned}
& B_{n}=\left(W_{N} e^{-N \partial_{s}} e^{n \partial_{s}}\left(W_{N} e^{-N \partial_{s}}\right)^{-1}\right)_{+}, \\
& C_{n}=\left(W_{N} e^{-n \partial_{s}}\left(W_{N}\right)^{-1}\right)_{-}, \\
& \mathcal{B}_{n}=\left(e^{\partial_{k_{n}}} W_{N} e^{\partial_{-k_{n}}} e^{-N \partial_{s}} e^{n \partial_{s}}\left(W_{N} e^{-N \partial_{s}}\right)^{-1}\right)_{+}, \\
& \mathcal{C}_{n}=\left(e^{\partial_{l_{n}}} W_{N} e^{-\partial_{l_{n}}} e^{n \partial_{s}}\left(W_{N}\right)^{-1}\right)_{-},
\end{aligned}
$$

and the coefficients of these operators are represented by using the Casoratitype determinants (20). If we set

$$
\begin{aligned}
W^{(\infty)} & =W_{N} e^{-N \partial_{s}}, \\
W^{(0)} & =W_{N},
\end{aligned}
$$

we can show that these operators $W^{(\infty)}$ and $W^{(0)}$ solve the Sato equations (8) and (12), as a consequence of Eqs. (22). 


\section{$\S 5 . \quad \tau$ Function and Hirota's Bilinear Equations}

Here we introduce a $\tau$ function which is a dependent variable in Hirota's bilinear equations. In this section, we reveal the $\tau$ function of the discrete analogue of the 2DTL hierarchy which is proposed in Section 3, and derive a part of infinitely many bilinear equations explicitly.

In the theory of the 2DTL hierarchy [19], it is known that the $\tau$ functions for the 2DTL hierarchy are defined through the functions $w_{j}^{(\infty)}$ and $w_{j}^{(0)}$. As we have shown in the preceding Section 3, the discrete analogue of the 2DTL hierarchy shares the difference operators $W^{(\infty)}, W^{(0)}$ with the continuous-time 2DTL hierarchy. The $\tau$ function for the discrete analogue of the 2DTL hierarchy depends on $s, x=\left(x_{1}, x_{2}, \ldots\right), y=\left(y_{1}, y_{2}, \ldots\right), k=\left(k_{1}, k_{2}, \ldots\right)$, and $l=\left(l_{1}, l_{2}, \ldots\right)$, and is also defined through the function

$$
\begin{aligned}
w_{j}^{(\infty)} & =\frac{p_{j}\left(-\widetilde{\partial_{x}}\right) \tau(s, x, y, k, l)}{\tau(s, x, y, k, l)}, \\
w_{j}^{(0)} & =\frac{p_{j}\left(-\widetilde{\partial_{y}}\right) \tau(s+1, x, y, k, l)}{\tau(s, x, y, k, l)},
\end{aligned}
$$

where $p_{j}, j=1,2, \ldots$, are polynomials defined by

$$
\exp \left(\sum_{n=1}^{\infty} t_{n} \lambda^{n}\right)=\sum_{j=0}^{\infty} p_{j}\left(t_{1}, t_{2}, \ldots\right) \lambda^{j},
$$

and $\widetilde{\partial_{x}}, \widetilde{\partial_{y}}$ are differential operators given by

$$
\begin{aligned}
& \widetilde{\partial_{x}}=\left(\frac{\partial}{\partial x_{1}}, \frac{1}{2} \frac{\partial}{\partial x_{2}}, \frac{1}{3} \frac{\partial}{\partial x_{3}}, \ldots\right), \\
& \widetilde{\partial_{y}}=\left(\frac{\partial}{\partial y_{1}}, \frac{1}{2} \frac{\partial}{\partial y_{2}}, \frac{1}{3} \frac{\partial}{\partial y_{3}}, \ldots\right) .
\end{aligned}
$$

Moreover, it is known that the difference operator $W^{(\infty)-1}$ and $W^{(0)-1}$ are written in terms of the $\tau$ functions as

$$
\begin{aligned}
W^{(\infty)-1} & =1+e^{-\partial_{s}} w_{1}^{(\infty) *}(s+1)+e^{-2 \partial_{s}} w_{2}^{(\infty) *}(s+1)+\cdots, \\
W^{(0)-1} & =w_{0}^{(0) *}(s+1)+e^{\partial_{s}} w_{1}^{(0) *}(s+1)+e^{2 \partial_{s}} w_{2}^{(0) *}(s+1)+\cdots,
\end{aligned}
$$

where

$$
\begin{aligned}
w_{j}^{(\infty) *} & =\frac{p_{j}\left(\widetilde{\partial_{x}}\right) \tau(s, x, y, k, l)}{\tau(s, x, y, k, l)}, \\
w_{j}^{(0) *} & =\frac{p_{j}\left(\widetilde{\partial_{y}}\right) \tau(s-1, x, y, k, l)}{\tau(s, x, y, k, l)} .
\end{aligned}
$$


From Eqs. (12) we have

$$
\begin{aligned}
\mathcal{B}_{n} & =\left(\Delta_{k_{n}}\left(W^{(\infty)}\right)+e^{\partial_{k_{n}}} W^{(\infty)} e^{-\partial_{k_{n}}} e^{n \partial_{s}}\right) W^{(\infty)-1} \\
& =\left(\Delta_{k_{n}}\left(W^{(0)}\right)+e^{\partial_{k_{n}}} W^{(0)} e^{-\partial_{k_{n}}} e^{n \partial_{s}}\right) W^{(0)-1} \\
\mathcal{C}_{n} & =\left(\Delta_{l_{n}}\left(W^{(\infty)}\right)+e^{\partial_{l_{n}}} W^{(\infty)} e^{-\partial_{l_{n}}} e^{-n \partial_{s}}\right) W^{(\infty)-1} \\
& =\left(\Delta_{l_{n}}\left(W^{(0)}\right)+e^{\partial_{l_{n}}} W^{(0)} e^{-\partial_{l_{n}}} e^{-n \partial_{s}}\right) W^{(0)-1}
\end{aligned}
$$

Hence we obtain the following relations

$$
\begin{aligned}
& \frac{1}{a_{n}} e^{\partial_{k_{n}}} W^{(\infty)} e^{-\partial_{k_{n}}} W^{(\infty)-1}+e^{\partial_{k_{n}}} W^{(\infty)} e^{-\partial_{k_{n}}} e^{n \partial_{s}} W^{(\infty)-1} \\
& =\frac{1}{a_{n}} e^{\partial_{k_{n}}} W^{(0)} e^{-\partial_{k_{n}}} W^{(0)-1}+e^{\partial_{k_{n}}} W^{(0)} e^{-\partial_{k_{n}}} e^{n \partial_{s}} W^{(0)-1}, \\
& \frac{1}{b_{n}} e^{\partial_{l_{n}}} W^{(\infty)} e^{-\partial_{l_{n}}} W^{(\infty)-1}+e^{\partial_{l_{n}}} W^{(\infty)} e^{-\partial_{l_{n}}} e^{-n \partial_{s}} W^{(\infty)-1} \\
& =\frac{1}{b_{n}} e^{\partial_{l_{n}}} W^{(0)} e^{-\partial_{l_{n}}} W^{(0)-1}+e^{\partial_{l_{n}}} W^{(0)} e^{-\partial_{l_{n}}} e^{-n \partial_{s}} W^{(0)-1},
\end{aligned}
$$

which are generating relations of infinite number of bilinear equations. From the definition of the $D$ operator (3), we have the following formula

$$
\sum_{i+j=k} p_{i}\left(-\widetilde{\partial}_{x}\right) u(x) \cdot p_{j}\left(\widetilde{\partial}_{x}\right) v(x)=p_{k}\left(-\widetilde{D}_{x}\right) u(x) \cdot v(x)
$$

where $\widetilde{D}_{x}, \widetilde{D}_{y}$ are defined by

$$
\widetilde{D}_{x}=\left(D_{x_{1}}, \frac{1}{2} D_{x_{2}}, \frac{1}{3} D_{x_{3}}, \ldots\right), \quad \widetilde{D}_{y}=\left(D_{y_{1}}, \frac{1}{2} D_{y_{2}}, \frac{1}{3} D_{y_{3}}, \ldots\right)
$$

Hereafter the unshifted independent variables of the $\tau$ function are often omitted for simplicity and only the shifted variables are written down explicitly. For example, $e^{\partial_{k_{n}}} \tau(s, x, y, k, l)$ and $e^{\partial_{l_{n}}} \tau(s, x, y, k, l)$ are written as $\tau\left(s ; k_{n}+1\right)$ 
and $\tau\left(s ; l_{n}+1\right)$, respectively. Substituting Eqs. (6) into (30a), we have

1.h.s. of (30a)

$$
\begin{aligned}
= & \left(\sum_{i=0}^{\infty} \frac{p_{i}\left(-\widetilde{\partial}_{x}\right) \tau\left(s ; k_{n}+1\right)}{\tau\left(s ; k_{n}+1\right)} e^{-i \partial_{s}}\right)\left(\frac{1}{a_{n}} \sum_{j=0}^{\infty} e^{-j \partial_{s}} \frac{p_{j}\left(\widetilde{\partial}_{x}\right) \tau\left(s+1 ; k_{n}\right)}{\tau\left(s+1 ; k_{n}\right)}\right) \\
& +\left(\sum_{i=0}^{\infty} \frac{p_{i}\left(-\widetilde{\partial}_{x}\right) \tau\left(s ; k_{n}+1\right)}{\tau\left(s ; k_{n}+1\right)} e^{-i \partial_{s}}\right) e^{n \partial_{s}}\left(\sum_{j=0}^{\infty} e^{-j \partial_{s}} \frac{p_{j}\left(\widetilde{\partial}_{x}\right) \tau\left(s+1 ; k_{n}\right)}{\tau\left(s+1 ; k_{n}\right)}\right) \\
= & \frac{1}{a_{n}} \sum_{i, j=0}^{\infty} \frac{p_{i}\left(-\widetilde{\partial}_{x}\right) \tau\left(s ; k_{n}+1\right) \cdot p_{j}\left(\widetilde{\partial}_{x}\right) \tau\left(s-i-j+1 ; k_{n}\right)}{\tau\left(s ; k_{n}+1\right) \tau\left(s-i-j+1 ; k_{n}\right)} e^{-(i+j) \partial_{s}} \\
& +\sum_{i, j=0}^{\infty} \frac{p_{i}\left(-\widetilde{\partial}_{x}\right) \tau\left(s ; k_{n}+1\right) \cdot p_{j}\left(\widetilde{\partial}_{x}\right) \tau\left(s-i-j+n+1 ; k_{n}\right)}{\tau\left(s ; k_{n}+1\right) \tau\left(s-i-j+n+1 ; k_{n}\right)} e^{(n-i-j) \partial_{s}},
\end{aligned}
$$

and

r.h.s. of (30a)

$$
\begin{aligned}
= & \left(\sum_{i=0}^{\infty} \frac{p_{i}\left(-\widetilde{\partial}_{y}\right) \tau\left(s+1 ; k_{n}+1\right)}{\tau\left(s ; k_{n}+1\right)} e^{i \partial_{s}}\right)\left(\frac{1}{a_{n}} \sum_{j=0}^{\infty} e^{j \partial_{s}} \frac{p_{j}\left(\widetilde{\partial}_{y}\right) \tau\left(s ; k_{n}\right)}{\tau\left(s+1 ; k_{n}\right)}\right) \\
& +\left(\sum_{i=0}^{\infty} \frac{p_{i}\left(-\widetilde{\partial}_{y}\right) \tau\left(s+1 ; k_{n}+1\right)}{\tau\left(s ; k_{n}+1\right)} e^{i \partial_{s}}\right) e^{n \partial_{s}}\left(\sum_{j=0}^{\infty} e^{j \partial_{s}} \frac{p_{j}\left(\widetilde{\partial}_{y}\right) \tau\left(s ; k_{n}\right)}{\tau\left(s+1 ; k_{n}\right)}\right) \\
= & \frac{1}{a_{n}} \sum_{i, j=0}^{\infty} \frac{p_{i}\left(-\widetilde{\partial}_{y}\right) \tau\left(s+1 ; k_{n}+1\right) \cdot p_{j}\left(\widetilde{\partial}_{y}\right) \tau\left(s+i+j ; k_{n}\right)}{\tau\left(s ; k_{n}+1\right) \tau\left(s+i+j+1 ; k_{n}\right)} e^{(i+j) \partial_{s}} \\
& +\sum_{i, j=0}^{\infty} \frac{p_{i}\left(-\widetilde{\partial}_{y}\right) \tau\left(s+1 ; k_{n}+1\right) \cdot p_{j}\left(\widetilde{\partial}_{y}\right) \tau\left(s+i+j+n ; k_{n}\right)}{\tau\left(s ; k_{n}+1\right) \tau\left(s+i+j+n+1 ; k_{n}\right)} e^{(i+j+n) \partial_{s}} .
\end{aligned}
$$

Thus from the $m$-th coefficients of (30a), we have

$$
\begin{aligned}
& \frac{1}{a_{n}} \sum_{i+j=-m \geq 0} \frac{p_{i}\left(-\widetilde{\partial}_{x}\right) \tau\left(s ; k_{n}+1\right) \cdot p_{j}\left(\widetilde{\partial}_{x}\right) \tau\left(s+m+1 ; k_{n}\right)}{\tau\left(s ; k_{n}+1\right) \tau\left(s+m+1 ; k_{n}\right)} \\
& \quad+\sum_{i+j=n-m \geq 0} \frac{p_{i}\left(-\widetilde{\partial}_{x}\right) \tau\left(s ; k_{n}+1\right) \cdot p_{j}\left(\widetilde{\partial}_{x}\right) \tau\left(s+m+1 ; k_{n}\right)}{\tau\left(s ; k_{n}+1\right) \tau\left(s+m+1 ; k_{n}\right)} \\
& =\frac{1}{a_{n}} \sum_{i+j=m \geq 0} \frac{p_{i}\left(-\widetilde{\partial}_{y}\right) \tau\left(s+1 ; k_{n}+1\right) \cdot p_{j}\left(\widetilde{\partial}_{y}\right) \tau\left(s+m ; k_{n}\right)}{\tau\left(s ; k_{n}+1\right) \tau\left(s+m+1 ; k_{n}\right)} \\
& \quad+\sum_{i+j=m-n \geq 0} \frac{p_{i}\left(-\widetilde{\partial}_{y}\right) \tau\left(s+1 ; k_{n}+1\right) \cdot p_{j}\left(\widetilde{\partial}_{y}\right) \tau\left(s+m ; k_{n}\right)}{\tau\left(s ; k_{n}+1\right) \tau\left(s+m+1 ; k_{n}\right)}
\end{aligned}
$$


or equivalently, a system of bilinear equations

$$
\left\{\begin{array}{rr}
\left(p_{-m}\left(-\widetilde{D}_{x}\right)+a_{n} p_{n-m}\left(-\widetilde{D}_{x}\right)\right) \tau\left(s ; k_{n}+1\right) \cdot \tau\left(s+m+1 ; k_{n}\right) & -\infty<m<0 \\
=0, & \\
\left(a_{n} p_{n}\left(-\widetilde{D}_{x}\right)+1\right) \tau\left(s ; k_{n}+1\right) \cdot \tau\left(s+1 ; k_{n}\right) & \\
\quad=\tau\left(s+1 ; k_{n}+1\right) \tau\left(s ; k_{n}\right), & \\
a_{n} p_{n-m}\left(-\widetilde{D}_{x}\right) \tau\left(s ; k_{n}+1\right) \cdot \tau\left(s+m+1 ; k_{n}\right) & \\
\quad=p_{m}\left(-\widetilde{D}_{y}\right) \tau\left(s+1 ; k_{n}+1\right) \cdot \tau\left(s+m ; k_{n}\right), & 0<m<n \\
a_{n} \tau\left(s ; k_{n}+1\right) \tau\left(s+n+1 ; k_{n}\right) & \\
=\left(p_{n}\left(-\widetilde{D}_{y}\right)+a_{n}\right) \tau\left(s+1 ; k_{n}+1\right) \cdot \tau\left(s+n ; k_{n}\right), & m=n \\
\left(p_{m}\left(-\widetilde{D}_{y}\right)+a_{n} p_{m-n}\left(-\widetilde{D}_{y}\right)\right) \tau\left(s+1 ; k_{n}+1\right) \cdot \tau\left(s+m ; k_{n}\right) & n<m<\infty \\
=0, &
\end{array}\right.
$$

for $n=1,2, \ldots, m=\ldots,-1,0,1,2, \ldots$, where we have used Eq. (31). Similarly, we obtain the other infinite number of bilinear difference equations from (30b)

$$
\left\{\begin{array}{lc}
\left(p_{-m}\left(-\widetilde{D}_{x}\right)+b_{n} p_{-n-m}\left(-\widetilde{D}_{x}\right)\right) \tau\left(s ; l_{n}+1\right) \cdot \tau\left(s+m+1 ; l_{n}\right) & \\
=0, & \\
\left(p_{n}\left(-\widetilde{D}_{x}\right)+b_{n}\right) \tau\left(s ; l_{n}+1\right) \cdot \tau\left(s-n+1 ; l_{n}\right) & \\
=b_{n} \tau\left(s+1 ; l_{n}+1\right) \tau\left(s-n ; l_{n}\right), & \\
p_{-m}\left(-\widetilde{D}_{x}\right) \tau\left(s ; l_{n}+1\right) \cdot \tau\left(s+m+1 ; l_{n}\right) & \\
\quad=b_{n} p_{m+n}\left(-\widetilde{D}_{y}\right) \tau\left(s+1 ; l_{n}+1\right) \cdot \tau\left(s+m ; l_{n}\right), & -n<m<0 \\
\tau\left(s ; l_{n}+1\right) \tau\left(s+1 ; l_{n}\right) & \\
=\left(b_{n} p_{n}\left(-\widetilde{D}_{y}\right)+1\right) \tau\left(s+1 ; l_{n}+1\right) \cdot \tau\left(s ; l_{n}\right), & m=0 \\
\left(p_{m}\left(-\widetilde{D}_{y}\right)+b_{n} p_{m+n}\left(-\widetilde{D}_{y}\right)\right) \tau\left(s+1 ; l_{n}+1\right) \cdot \tau\left(s+m ; l_{n}\right) & 0<m<\infty \\
=0 &
\end{array}\right.
$$

for $n=1,2, \ldots, m=\ldots,-1,0,1,2, \ldots$ Eqs. (32) and (33) are the bilinear equations of the discrete analogue of the 2DTL hierarchy. Starting from these bilinear equations, we can derive the discrete equations. For example, from Eq. (32) by setting $m=0, n=1$

$$
\left(-a_{1} D_{x_{1}}+1\right) \tau\left(s ; k_{1}+1\right) \cdot \tau\left(s+1 ; k_{1}\right)=\tau\left(s+1 ; k_{1}+1\right) \tau\left(s ; k_{1}\right)
$$

and Eq. (33) by setting $m=-1, n=1$

$$
\left(-D_{x_{1}}+b_{1}\right) \tau\left(s ; l_{1}+1\right) \cdot \tau\left(s ; l_{1}\right)=b_{1} \tau\left(s+1 ; l_{1}+1\right) \tau\left(s-1 ; l_{1}\right)
$$

we obtain the discrete 2DTL equation (17). Here we set the dependent variables 
$u_{1}^{(1)}, v_{0}^{(1)}$ as

$$
\begin{aligned}
& u_{1}^{(1)}=w_{1}^{(\infty)}\left(s ; k_{1}+1, l_{1}\right)+w_{1}^{(\infty) *}\left(s+1 ; k_{1}, l_{1}\right), \\
& v_{0}^{(1)}=w_{0}^{(0)}\left(s ; k_{1}, l_{1}+1\right) w_{0}^{(0) *}\left(s ; k_{1}, l_{1}\right),
\end{aligned}
$$

which are determined by the definition (11).

\section{§6. Matrix Form of Discrete 2DTL Hierarchy}

We have developed a theory of a discrete analogue of the 2DTL hierarchy by operator formalism. In Section 3 we have proposed three types of expressions (12), (13) and (14) for the discrete analogue of the 2DTL hierarchy. Those expressions are slightly complicated, so we give another expression by using matrix forms.

First we rewrite the discrete Zakharov-Shabat equations (14) by using difference operators $\mathcal{B}_{n}$ and $\mathcal{C}_{n}$ as

$$
\begin{aligned}
(1+ & \left.a_{n} \mathcal{B}_{n}\left(k_{m}+1, k_{n}\right)\right)\left(1+a_{m} \mathcal{B}_{m}\left(k_{m}, k_{n}\right)\right) \\
\quad & =\left(1+a_{m} \mathcal{B}_{m}\left(k_{m}, k_{n}+1\right)\right)\left(1+a_{n} \mathcal{B}_{n}\left(k_{m}, k_{n}\right)\right) \\
(1+ & \left.b_{n} \mathcal{C}_{n}\left(l_{m}+1, l_{n}\right)\right)\left(1+b_{m} \mathcal{C}_{m}\left(l_{m}, l_{n}\right)\right) \\
\quad & =\left(1+b_{m} \mathcal{C}_{m}\left(l_{m}, l_{n}+1\right)\right)\left(1+b_{n} \mathcal{C}_{n}\left(l_{m}, l_{n}\right)\right) \\
(1+ & \left.a_{n} \mathcal{B}_{n}\left(k_{n}, l_{m}+1\right)\right)\left(1+b_{m} \mathcal{C}_{m}\left(k_{n}, l_{m}\right)\right) \\
\quad & =\left(1+b_{m} \mathcal{C}_{m}\left(k_{n}+1, l_{m}\right)\right)\left(1+a_{n} \mathcal{B}_{n}\left(k_{n}, l_{m}\right)\right)
\end{aligned}
$$

The discrete analogue of the 2DTL hierarchy can be formulated by infinite matrices $R_{n}, L_{n}$ which are equivalent to the difference operators $\left(1+a_{n} \mathcal{B}_{n}\right)$ and $\left(1+b_{m} \mathcal{C}_{m}\right)$, respectively. Let us introduce

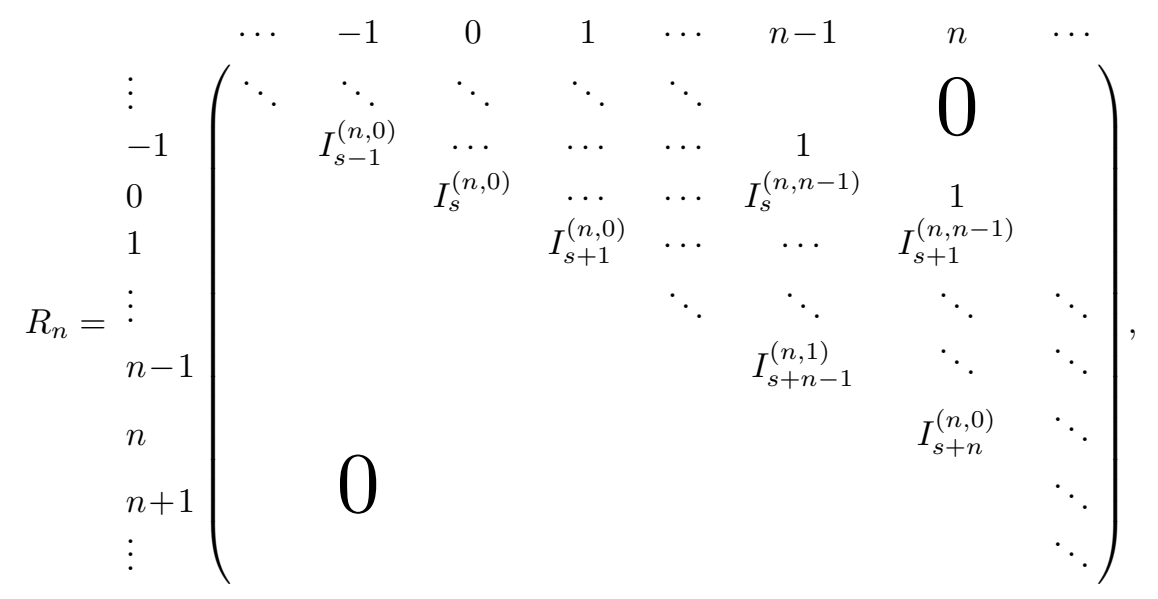




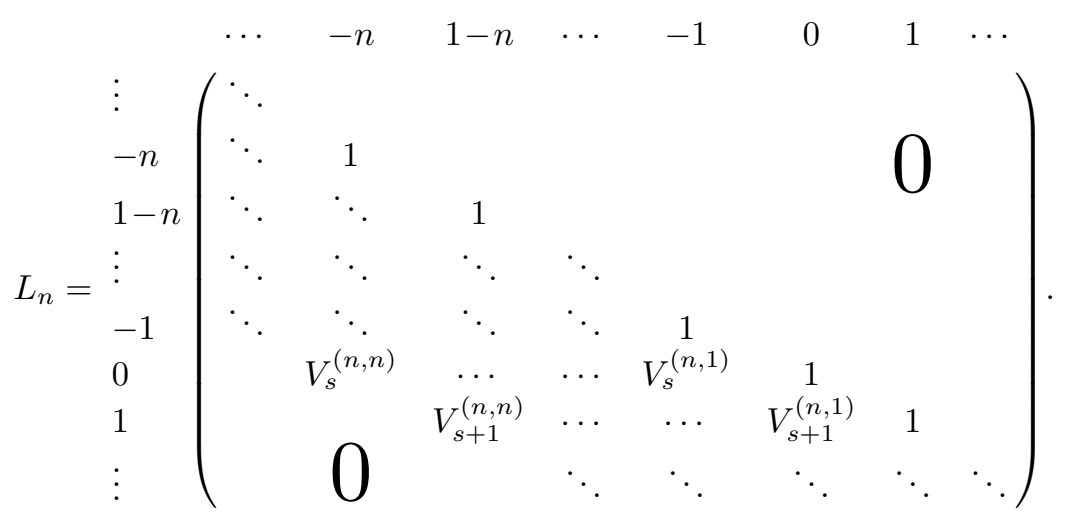

Here the dependent variables $I_{s}^{(n, 0)}, I_{s}^{(n, 1)}, I_{s}^{(n, 2)}, \ldots, V_{s}^{(n, 1)}, V_{s}^{(n, 2)}, \ldots$ are given by

$$
\begin{array}{rlrl}
I_{s}^{(n, 0)} & =1+a_{n} u_{n}^{(n)}(s), & I_{s}^{(n, 1)}=a_{n} u_{n-1}^{(n)}(s), \\
I_{s}^{(n, 2)}=a_{n} u_{n-2}^{(n)}(s), & \cdots, \\
V_{s}^{(n, 1)}=b_{n} v_{n-1}^{(n)}(s), & V_{s}^{(n, 2)}=b_{n} v_{n-2}^{(n)}(s), \\
V_{s}^{(n, 3)}=b_{n} v_{n-3}^{(n)}(s), & \cdots .
\end{array}
$$

The products infinite matrices $L_{m} R_{n}$ and $R_{m} L_{n}$ for $m, n=1,2, \ldots$ are welldefined. See Ueno-Takasaki [19], p. 4.

Then the discrete analogue of the 2DTL hierarchy (13) or (14) can be written in the following discrete Lax forms

$$
\begin{aligned}
R_{m}\left(k_{m}, k_{n}+1\right) R_{n}\left(k_{m}, k_{n}\right) & =R_{n}\left(k_{m}+1, k_{n}\right) R_{m}\left(k_{m}, k_{n}\right), \\
L_{m}\left(l_{m}, l_{n}+1\right) L_{n}\left(l_{m}, l_{n}\right) & =L_{n}\left(l_{m}+1, l_{n}\right) L_{m}\left(l_{m}, l_{n}\right), \\
R_{n}\left(k_{m}+1, l_{n}\right) L_{m}\left(k_{m}, l_{n}\right) & =L_{m}\left(k_{m}, l_{n}+1\right) R_{n}\left(k_{m}, l_{n}\right),
\end{aligned}
$$

for $m, n=1,2, \ldots$, which is a generalization to infinite matrices of the LR factorization algorithm.

\section{$\S 7 . \quad$ Concluding Remarks}

In this paper, we have presented a discrete analogue of the 2DTL hierarchy by using the formulation of the continuous-time 2DTL hierarchy [19]. It has been shown that the subjects originated from the Sato theory are useful for the discrete-time integrable systems. The difference operators $W^{(\infty)}$ and $W^{(0)}$ introduced by Ueno-Takasaki [19] also play an important role in the discretetime analogue of the 2DTL hierarchy, likewise in the continuous-time 2DTL 
hierarchy. This framework might be applicable to other discrete-time soliton equations.

We have introduced an infinite number of discrete-time variables in the discrete Sato equation (12) which governs the discrete-time evolutions. Miwa [10] also introduced an infinite number of discrete-time variables of the discrete $\mathrm{KP}$ equation as we have seen in Introduction. There is an interesting contrast in the linear equations appeared in the special solutions of the Casorati determinant form. Namely, the orders of the linear equations (5) of the discrete KP equation are all same, while the orders of the linear equations (21c), (21d) of the discrete analogue of the 2DTL hierarchy are different from each other. We can introduce a more general case where the linear equations of the discrete analogue of the 2DTL hierarchy are given as

$$
\begin{aligned}
& \Delta_{\tilde{k}_{n}} f_{j}(s)=f_{j}(s+n)+\sum_{i=1}^{n-1} \alpha_{n i} f_{j}(s+n-i), \\
& \Delta_{\tilde{l}_{n}} f_{j}(s)=f_{j}(s-n)+\sum_{i=1}^{n-1} \beta_{n i} f_{j}(s-n+i),
\end{aligned}
$$

where $\widetilde{k}_{n}, \widetilde{l}_{n}, n=1,2, \ldots$ are new discrete variables, and $\alpha_{i j}, \beta_{i j}, i, j=1,2, \ldots$ are constants. In this case, we can derive a generalized discrete Sato equations

$$
\begin{aligned}
& \Delta_{\widetilde{k}_{n}}\left(W^{(\infty)}\right)=\widetilde{\mathcal{B}}_{n} W^{(\infty)}-e^{\partial_{\widetilde{k}_{n}} W^{(\infty)}} e^{-\partial_{\tilde{k}_{n}}}\left(e^{n \partial_{s}}+\sum_{i=1}^{n-1} \alpha_{n i} e^{(n-i) \partial_{s}}\right) \\
& \Delta_{\widetilde{l}_{n}}\left(W^{(\infty)}\right)=\widetilde{\mathcal{C}}_{n} W^{(\infty)}-e^{\partial_{\widetilde{l}_{n}} W^{(\infty)}} e^{-\partial_{\widetilde{l}_{n}}}\left(e^{-n \partial_{s}}+\sum_{i=1}^{n-1} \beta_{n i} e^{(i-n) \partial_{s}}\right) \\
& \Delta_{\tilde{k}_{n}}\left(W^{(0)}\right)=\widetilde{\mathcal{B}}_{n} W^{(0)}-e^{\partial_{\tilde{k}_{n}} W^{(0)}} e^{-\partial_{\tilde{k}_{n}}}\left(e^{n \partial_{s}}+\sum_{i=1}^{n-1} \alpha_{n i} e^{(n-i) \partial_{s}}\right) \\
& \Delta_{\widetilde{l}_{n}}\left(W^{(0)}\right)=\widetilde{\mathcal{C}}_{n} W^{(0)}-e^{\partial_{\tilde{l}_{n}} W^{(0)}} e^{-\partial_{\widetilde{l}_{n}}}\left(e^{-n \partial_{s}}+\sum_{i=1}^{n-1} \beta_{n i} e^{(i-n) \partial_{s}}\right)
\end{aligned}
$$

where the difference operators $\widetilde{\mathcal{B}}_{n}$ and $\widetilde{\mathcal{C}}_{n}$ are defined as

$$
\begin{aligned}
& \widetilde{\mathcal{B}}_{n}=e^{\partial_{\widetilde{k}_{n}} W^{(\infty)}} e^{-\partial_{\widetilde{k}_{n}}}\left(e^{n \partial_{s}}+\sum_{i=1}^{n-1} \alpha_{n i} e^{(n-i) \partial_{s}}\right) W^{(\infty)-1}, \\
& \widetilde{\mathcal{C}}_{n}=e^{\partial_{\widetilde{l}_{n}}} W^{(0)} e^{-\partial_{\widetilde{l}_{n}}}\left(e^{-n \partial_{s}}+\sum_{i=1}^{n-1} \beta_{n i} e^{(i-n) \partial_{s}}\right) W^{(0)-1}
\end{aligned}
$$


If we choose the constants $\alpha_{i j}, i, j=1,2, \ldots$ suitably, one can find the following relationship between the discrete KP hierarchy and the discrete analogue of the 2DTL hierarchy

$$
e^{-\partial_{\tilde{k}_{n}}} f(s)=e^{\partial_{m_{1}}+\partial_{m_{2}}+\cdots+\partial_{m_{n}}} f(s) .
$$

Thus each discrete variable $\widetilde{k}_{n}$ of the discrete analogue of the 2DTL hierarchy correspond to the unit of $n$ discrete variables $m_{1}, m_{2}, \ldots, m_{n}$ of the discrete KP hierarchy.

\section{References}

[1] Date, E., Jimbo, M., Kashiwara, M. and Miwa, T., Transformation groups for soliton equations, in Nonlinear Integrable Systems, (M. Jimbo and T. Miwa, eds.), World Scientific, Singapore, 1983, pp. 39-119.

[2] Date, E., Jimbo, M. and Miwa, T., Method for generating discrete soliton equations I, J. Phys. Soc. Japan, 51 (1982), 4116-4124.

[3] $\longrightarrow$, Method for generating discrete soliton equations II, J. Phys. Soc. Japan, 51 (1982), 4125-4131.

[4] - Method for generating discrete soliton equations III, J. Phys. Soc. Japan, 52 (1983), 388-393.

[5] $\longrightarrow$, Method for generating discrete soliton equations IV, J. Phys. Soc. Japan, 52 (1983), 761-765.

[6] $\_$Method for generating discrete soliton equations V, J. Phys. Soc. Japan, 52 (1983), 766-771.

[7] Hirota, R., Nonlinear partial difference equations. II. Discrete-time Toda equation, J. Phys. Soc. Japan, 43 (1977), 2074-2078.

[8] - Discrete analogue of a generalized Toda equation, J. Phys. Soc. Japan, 50 (1981), 3785-3791.

[9] Hirota, R., Tsujimoto, S. and Imai, T., Difference Scheme of Soliton Equations, in Future Directions of Nonlinear Dynamics in Physical and Biological Systems, (P. L. Christiansen, J. C. Eilbeck and R. D. Parmentier, eds.), 312 in NATO ASI Ser. B, Physics, Plenum Press, New York, 1993, pp.7-15.

[10] Miwa, T., On Hirota's difference equations, Proc. Japan Acad., 58 (1982), 9-12.

[11] Ohta, Y., Satsuma, J., Takahashi, D. and Tokihiro, T., An elementary introduction to Sato theory, Prog. Theor. Phys. Suppl., 94 (1988), 210-241.

[12] Papageorgiou, V., Grammaticos, B. and Ramani, A., Orthogonal polynomial approach to discrete Lax-pairs for initial boundary-value problems of the QD algorithm, Lett. Math. Phys., 34 (1995), 91-101.

[13] Sato, M., Soliton equations as dynamical systems on infinite dimensional Grassmann manifolds, RIMS, Kyoto Univ. Kokyuroku, 439 (1981), 30-46.

[14] - The KP hierarchy and infinite-dimensional Grassmann manifolds, in Proc. Symp. Pure Math., Theta functions, Bowdin 1987, (L. Ehrenpreis and R. C. Gunning, eds.), vol. 49-Part1, AMS, Providence, 1989, pp. 51-66.

[15] Sato, M. and Sato, Y., Soliton equations as dynamical systems on infinite dimensional Grassmann manifold, in Nonlinear PDE in Applied Science, Proc. U. S.-Japan Seminar, Tokyo 1982, Kinokuniya, Tokyo, 1983, pp. 259-271.

[16] Suris, Yu. B., Discrete-time generalized Toda lattices: complete integrability and relations with relativistic Toda lattices, Phys. Lett. A, 145 (1990), 113-119. 
[17] Suris, Yu. B., Bi-Hamiltonian structure of the qd algorithm and new discretizations of the Toda lattice, Phys. Lett. A, 206 (1995), 153-161.

[18] Tsujimoto, S., On exact solutions of discrete soliton equations, RIMS, Kyoto Univ. Kokyuroku, 1005 (1997), 28-36 (in Japanese).

[19] Ueno, K. and Takasaki, K., The Toda lattice hierarchy, in Group Representations and Systems of Differential Equations, Adv. Stud. Pure Math., 4, Kinokuniya, Tokyo, 1984, pp. 1-139. 